\title{
Detecting emotions through non-invasive wearables
}

\author{
J. A. Rincon, V. Julian, C. Carrascosa \\ Universitat Politècnica de València. D. Sistemas Informáticos y Computación \\ \{jrincon,vinglada, carrasco@dsic.upv.es\} \\ A. Costa, P. Novais \\ Centro ALGORITMI, Escola de Engenharia, Universidade do Minho, Guimarães \\ \{acosta,pjon@di.uminho.pt\}
}

\begin{abstract}
Current research on computational intelligence is being conducted in order to emulate and/or detect emotional states using specific devices such as wristbands or similar wearables. In this sense, this paper proposes the use of intelligent wristbands for the automatic detection of emotional states in order to develop an application which allows to extract, analyze, represent and manage the social emotion of a group of entities. Nowadays, most of the existing approaches are centered in the emotion detection and management of a single entity. The designed system has been developed as a multi-agent system where each agent controls a wearable device and is in charge of detecting individual emotions based on bio-signals.

Keywords: Multi-agent Systems; Wearables ; Emotion detection
\end{abstract}

\section{Introduction}

The emulation of emotional states allow machines to represent some human emotions. Current research on computational intelligence is being conducted in order to emulate and/or detect those emotional states [1]. This artificial represen-

5 tation of emotions is being used by machines to improve the interaction process with humans. In order to create a fluid emotional communication between human and machines, the machines need first to detect the emotion of the human with the final purpose of enhancing decison-making processes while improving humancomputer interactions [2]. In order to accomplish with these tasks, it is necessary to 
body gestures, and biosignals.

Human beings perceive and analyse a wide range of stimuli in different environments. These stimuli interfere in our commodity levels modifying our emotional states. Before each one of these stimuli, humans generate several type of responses, varying our face gestures, body movement or bio-electrical impulses. These variations in our emotional states could be used as a very useful information for machines. To do this, machines will require the capability of interpreting correctly such variations. This is the reason for the design of emotional models that interpret and represent the different emotions in a computational way. In this case, emotional models such as Ortony, Clore \& Collins model [3] and the PAD (PleasureArousal-Dominance) model [4] are the most used ones to detect or simulate emotional states. With the emergence of new smart devices, in areas such as ubiquitous computation and ambient intelligent, emotional states are now a very valuable information, which allows the development of new applications that help to improve the human being life quality in a more accurate and reliable way.

Nevertheless, for the time being the detection of the joined emotion of an heterogeneous group of people is still an open issue. Most of the existing approaches are centered in the emotion detection and management of a single entity. In this work we propose to detect the social emotion of groups of people in an Ambient Intelligence (AmI) application with the help of smart wearables. Specifically, we propose a system that controls automatically the detection of the emotions of the people with the use of individual wristbands. Thus, the main goal of the proposed system is to obtain the social emotion of this group of people in order to try to improve the well-being of that people (as an example, play some kind of music to increase happiness of the people). Each one of the individuals will have a, possibly different, emotional response. This response will be detected and transmitted by the wristbands in order to calculate a social emotion of the set of individuals. This social emotion will be used to predict the most appropriated action according to the domain where the application is used. In this way, the designed system has been developed as a multi-agent system where each agent controls a wearable device 
and is in charge of detecting individual emotions based on bio-signals.

The rest of the paper is structured as follows: section 2 gives an overview of the related state of the art; section 3 describes the system focusing on the description of the wristband agent; finally, section 4 presents some conclusions.

\section{2. State of the Art}

The social issues that affect society strongly push the interest in solutions that aim to solve those issues. AmI is gaining traction due to its usage in complex environments to help elderly people sustain an independent and active life. AmI projects help in providing day-care through monitoring fragile people (like elderly or mentally/physically challenged) and acting through traditional interfaces (sound, screens, etc.) or changing environment conditions and objects (through actuators or smart objects). This means that a home equipped with these devices and services would improve the people lives and, at the same time, be cost-effective. These systems require an initial significant investment (the cost of the devices and the installation),

55 but they have a low maintenance cost, thus improving their cost on the long term. Despite the several aims AmI projects have, they can be clustered in five clusters of operational areas [5]: Daily living activities, Fall and movement detection, Location tracking, Medication control, Medical status monitoring.

The clustering is important as it classifies the contained projects and provide focus to an extensive area. Some are contained in an cluster and some spread across various, but most are cantered in a cluster. Next we present a project for each of these areas, providing a sample of the numerous existing projects. This representation is important as we are able to classify our project and show the common and uncommon features that it shares with the represented projects.

Daily living activities: [6] presents a platform to identify the users actions (moving around and interacting with objects) with RFID technology. They have a database with pre-trained values that correlate to the RFID signals, and a fast inference mechanism that allows the identifications of the actions within a given space. One of the issues of this platform is the limited range of the RFID beacons, 
which require that the people that use the tags to be close to them, resulting in an unnatural interaction with the platform.

Fall and movement detection: in terms of hardware, the projects in this area resort to the following (one or a mixture of them): cameras, accelerometers and pressure sensors. [7] presents a platform that uses cameras (to register the visual information) and then process that data to provide accurate results. This platform does not requires direct interaction from the users but are very invasive in terms of privacy. The cameras have to be placed at critical places, like the bathroom, a place where privacy is of the uttermost importance and the law impedes the placement of cameras. Thus, this platform (and similar ones) are very limited in terms of functionality (by being limited to some areas of the house).

Location tracking: [8] presents a location tracking system that uses a smartphone sensors to provide information about the current location of the system users to other AmI systems. This information can then be proceeded to informal and formal caregivers and medical personnel. These type of systems require the constant monitoring of the localization, thus there is no guarantee of privacy. This intrusion is not done directly but by allowing 3rd party users to constantly know the location of another person the system becomes very intrusive [9]. Moreover, the drainage of battery is still an important drawback, and localization consumes a large amount of battery and carry more batteries is not always feasible.

Medication control: these projects consist in systems that help the users to remind the medications that they have to take $[10,11]$. They play an important role on the users life, as a large number of elderly people have trouble in remembering to take their medication. These systems are mostly recommenders and can only provide simple information. These type of applications are commonly available for mobile devices and desktop computers.

Medical status monitoring: [12, 13, 14] present platforms that are constituted by sensor systems that are in direct contact with the human body. These sensor systems create a body area network and provide information about the users vital sign and bodily functions. For instance, all three works presented capture electrocardiograms. [13] uses the electrocardiogram information and ballistocardiogram infor- 
mation to assert if vehicle drivers are calm and concentrated or if they are stressed or having some kind of medical issue (the project is directed to elderly people). [14] presents a system that manages home lights (brightness and colour) according to the users physical state. The users carry a biosignal reading glove that sends

the readouts to the server and according to their pulse/heart-rate the lights are dimmed or colour changed. The glove has to be worn constantly and is limited to a confined range of actions (it cannot be wetted or used to manage heavy objects). The problem with these systems is that they require users to attach sensors on their own body (the case of [12]) or that the users are in a very controlled environment like [13].

One common problem revealed of these projects is that they are interested in developing their components but do not reveal any particular interest on the users opinion about those components. Only recently the topic of invasiveness has been studied due to the high reluctance of the users using clumsy and complex apparatus [15]. Our goal is to produce a system that increases the comfort level of the users through the use of a non-invasive wearable bracelet that performs body monitoring to attain the users' emotional status.

\section{System Proposal}

This section explains the different elements that compose the multi-agent system, which describe a way to detect emotions based on bio-signals. One of the main problems to detect human emotions is the information capture, existing different ways to get this information. Normally, this information is acquired using image processing, but we can find other ways such as capturing and processing speech, body gesture and bio-signals. The emotion detection using bio-signals is focused in our body signals as skin resistance (GSR), photoplethysmogram, heart rate and electroencephalography. This paper only takes into account three of these signals, since acquiring electroencephalography signals needs a big amount of sensors connected to the user, so it could bother to him more than wearing a wristband.

In current years, the use of wearable devices has been growing, devices such as 

which will have the know-how of the specific domain. This agent uses this social

\footnotetext{
${ }^{1}$ http://www.samsung.com

${ }^{2}$ http://www.apple.com
} 
emotional value to calculate what is the next action to be done. After different executions, the Manager agent can evaluate the effect that the actions has had over

\footnotetext{
${ }^{3}$ http://www.csea.phhp.ufl.edu/Media.html
} 


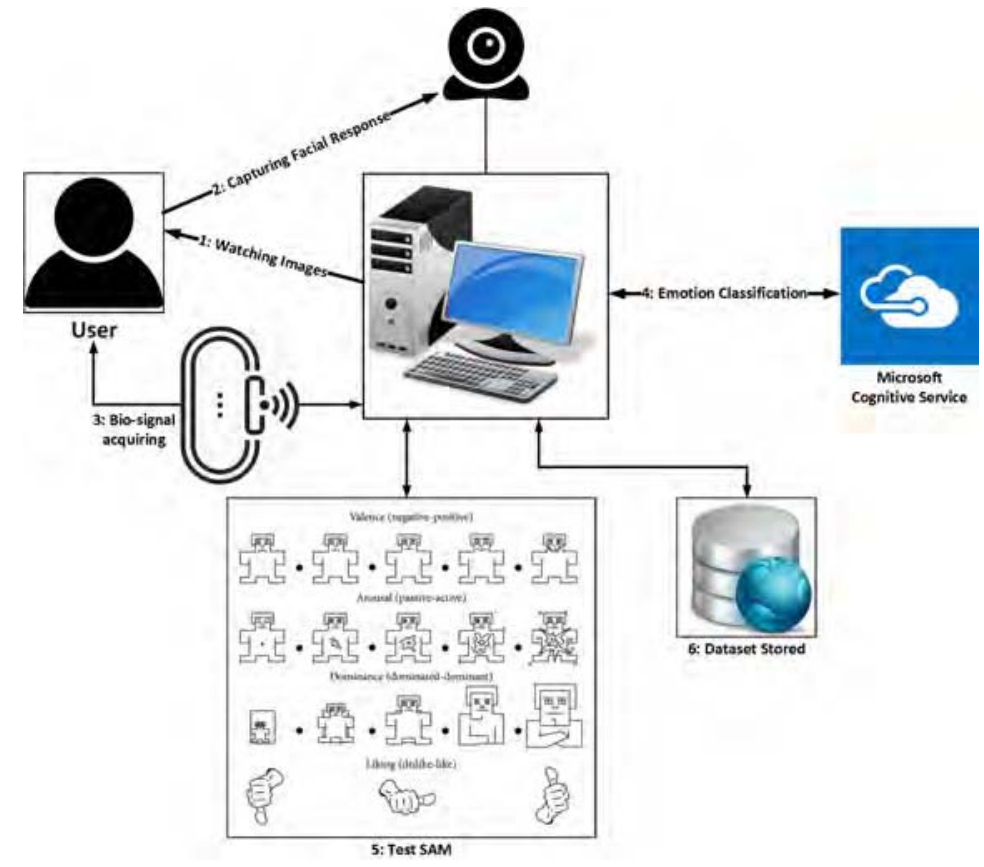

Figure 1: Graphical view of the proposed process.

service, the bio-signals acquired through the wristband and the SAM test result. This dataset is used to train the machine learning model that will be integrated into the wristband. The GUI (Figure 2) was divided in three sections one to show the image with which we want stimulate the emotional change. The second is the image captured using the web cam and the third is the SAM test.

The Figure 3 shows the wave form of a GSR signal (red line) and photoplethysmogram signal (blue line), acquired through the wristband.

\subsection{Data Acquisition Process}

This process made by the Wristband agent is responsible to capture the different needed bio-signals. To do this, the Wristband agent uses different sensors. The sensors used are: GSR and Photoplethysmogram (Figure4). The GSR measures the galvanic skin response. The measurement is performed by passing through the skin a very low current, and storing small variations in voltage. On the other hand, 


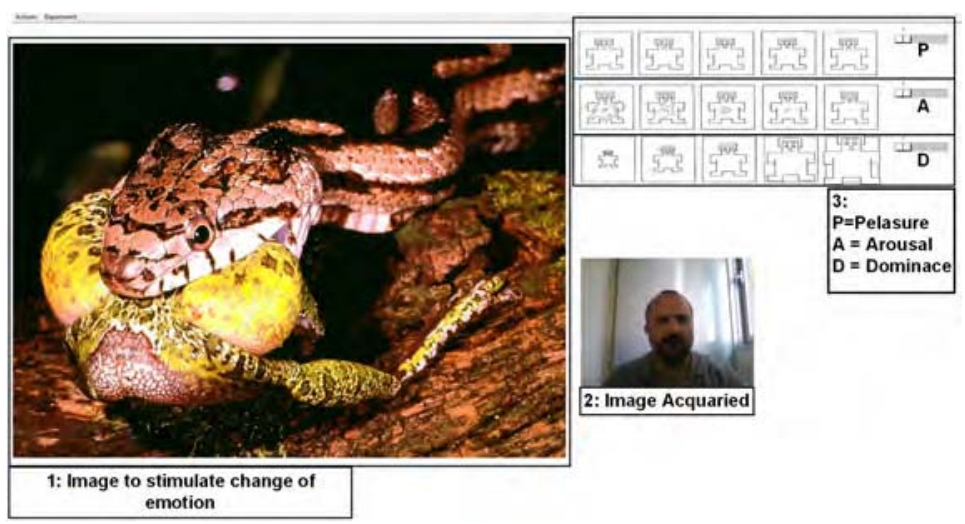

Figure 2: Graphical interface used to get the data.

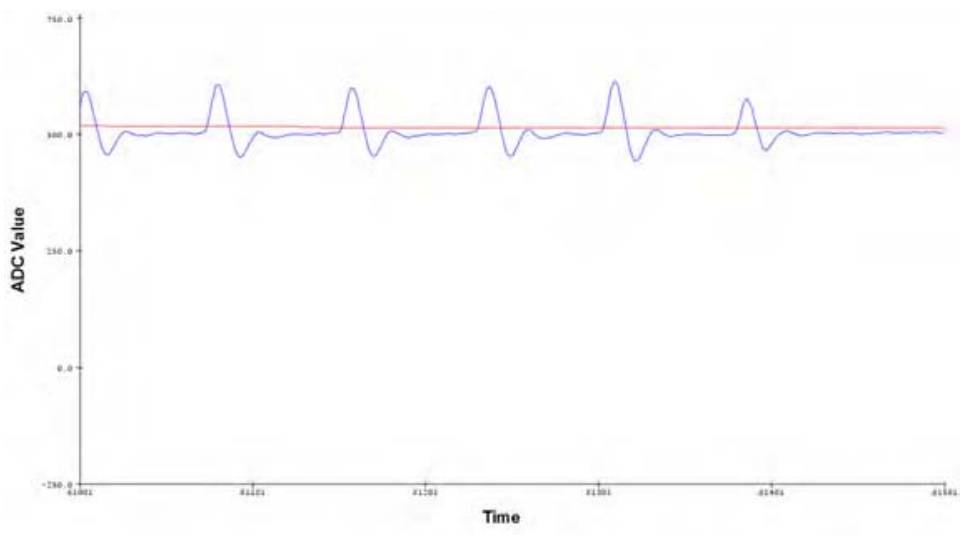

Figure 3: GSR Raw data.

the Photoplethysmogram is a process of applying a light source measuring the light reflected by the skin. The received signal consists of pulses that reflect the change in vascular blood volume with each cardiac beat. The information captured by each one of these sensors is subsequently preprocessed. This last process allows to convert the measure captured for each sensor in the corresponding units. The GSR sensor converts the measurement of the skin conductance in $\mathrm{Ohm}$ and the Photoplethysmogram returns raw data that can be easily processed. 


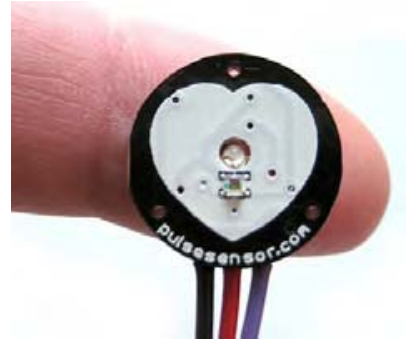

(a) Heart Rate Sensor

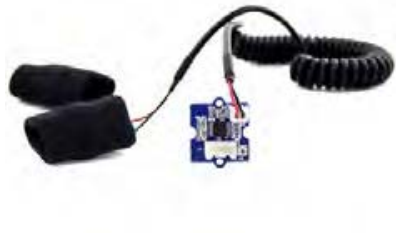

(b) Skin Resistance Sensor

Figure 4: View of the employed sensors.

\subsection{Emotion Classification}

In this section we describe the method to do the emotion recognition using the bio-signals acquired with the wristband. As commented in the previous section, we have made a dataset to train an ANN to be able to classify the user emotion from bio-signals captured by the wristband. This dataset has as inputs the three features corresponding to the three captured bio-signals: GSR, photoplethysmogram, and temperature as input. The dataset output is composed of the emotion detected using Microsoft cognitive service and the SAM test. Each input channel of the wristband is formed for 256 samples, each one of them is an input of our neural network. The neural network has also five hidden neurons and seven outputs (each output corresponds to an specific emotion). The architecture of our neural network is shown in Figure 5.

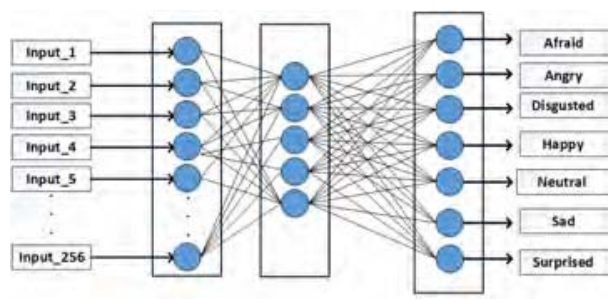

Figure 5: Neural Network Architecture.

The ANN has a background propagation architecture and was trained using a supervised methodology, since the objective of the network is to classify the human 

of the group.

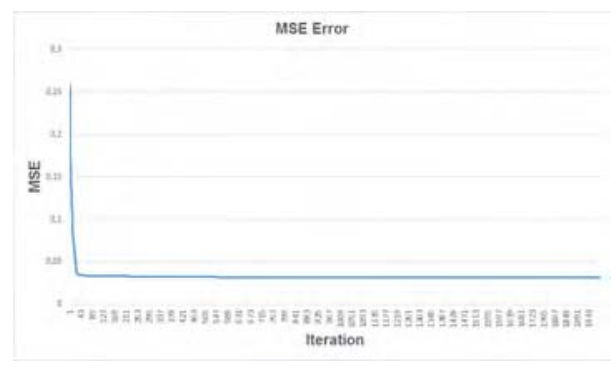

Figure 6: Mean square error of the neural network.

\subsection{Wristband Prototype}

This section describes the design of the physical wristband. This device was developed using an Arduino mini ${ }^{4}$ and is responsible for acquiring the GSR, PPG, Temperature and Acceleration, likewise it handles the communication with the in230 terface.

As before commented, the prototype has been designed as a wristband in which the Wristband Agent is deployed. Figure 7 shows the different components of our wristband prototype which are the following: (1) Power Supply and Battery Charger (3.7 volt battery); (2) Sensors: for acquisition of GSR and Photoplethysmogram 235 signals; and (3) Arduino mini processor. The bio-signals captured in the wristband are passed by an Analog to Digital Conversion or $A D C$ allowing the discretization of the analogue signals.

\footnotetext{
${ }^{4}$ https://store.arduino.cc/arduino-mini-05
} 


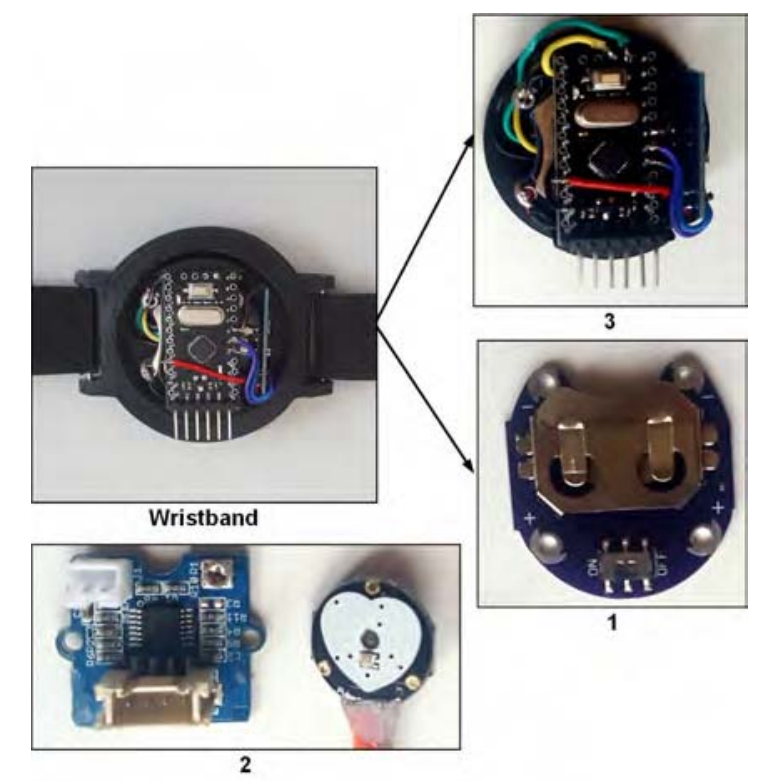

Figure 7: Components of the Smart Wristband Prototype.

\section{Conclusions and future work}

This paper presents how to integrate non-invasive biosignals for the detection of human emotional states through an agent-based application. The identification and detection of human emotional states allow the enhancement of the decision-making process of intelligent agents. The proposed application allows extracting (in a noninvasive way) the social emotion of a group of persons by means of wearables facilitating the decision-making in order to change the emotional state of the individuals.

245 As commented before, the application incorporates automatic emotion recognition using biosignals and machine learning techniques, which are easily included in the proposed system. The flexibility and dynamism of the proposed application allow the integration of new sensors or signals in future stages of the project. Moreover, as future work, we want to apply this system to other application domains, specifically the proposed framework fits with the industrial one, for instance representing production lines including the individuals and their emotional states as yet another elements to be considered in the production line. 


\section{Bibliography}

[1] J. Gratch, S. Marsella, Tears and fears: Modeling emotions and emotional behaviors in synthetic agents, in: Proceedings of the fifth international conference on Autonomous agents, ACM, 2001, pp. 278-285.

[2] C. Maaoui, a. Pruski, Emotion recognition through physiological signals for human-machine communication, Cutting Edge Robotics (2010) 317-333.

[3] B. N. Colby, A. Ortony, G. L. Clore, A. Collins, The Cognitive Structure of Emotions, Vol. 18, Cambridge University Press, 1989.

[4] A. Mehrabian, Analysis of affiliation-related traits in terms of the PAD Temperament Model., The Journal of psychology 131 (1) (1997) 101-117.

[5] H. Alemdar, C. Ersoy, Wireless sensor networks for healthcare: A survey, Computer Networks 54 (15) (2010) 2688-2710.

[6] K. P. Fishkin, B. Jiang, M. Philipose, S. Roy, I sense a disturbance in the force: Unobtrusive detection of interactions with RFID-tagged objects, in: UbiComp 2004: Ubiquitous Computing, Springer Berlin Heidelberg, 2004, pp. 268-282.

[7] J. C. Castillo, J. Serrano-Cuerda, A. Fernández-Caballero, A. MartínezRodrigo, Hierarchical architecture for robust people detection by fusion of infrared and visible video, in: Intelligent Distributed Computing IX, Springer Berlin Heidelberg, 2015, pp. 343-351.

[8] J. Ramos, T. Oliveira, K. Satoh, J. Neves, P. Novais, Orientation system based on speculative computation and trajectory mining, in: Highlights of Practical Applications of Scalable Multi-Agent Systems. The PAAMS Collection, Springer Nature, 2016, pp. 250-261.

[9] P. Hert, S. Gutwirth, A. Moscibroda, D. Wright, G. González Fuster, Legal safeguards for privacy and data protection in ambient intelligence, Personal and Ubiquitous Computing 13 (6) (2008) 435-444. 
[10] K. Stawarz, A. L. Cox, A. Blandford, Don't forget your pill!, in: Proceedings of the 32nd annual ACM conference on Human factors in computing systems CHI'14, Association for Computing Machinery (ACM), 2014.

[11] N. Tran, J. M. Coffman, K. Sumino, M. D. Cabana, Patient reminder systems and asthma medication adherence: a systematic review, Journal of Asthma 51 (5) (2014) 536-543.

[12] A. Hristoskova, V. Sakkalis, G. Zacharioudakis, M. Tsiknakis, F. D. Turck, Ontology-driven monitoring of patient's vital signs enabling personalized medical detection and alert, Sensors 14 (1) (2014) 1598-1628.

[13] M. Walter, B. Eilebrecht, T. Wartzek, S. Leonhardt, The smart car seat: personalized monitoring of vital signs in automotive applications, Pers Ubiquit Comput 15 (7) (2011) 707-715.

[14] E. Maier, G. Kempter, ALADIN - a magic lamp for the elderly?, in: Handbook of Ambient Intelligence and Smart Environments, Springer Berlin Heidelberg, 2010, pp. 1201-1227.

[15] R. Fensli, P. E. Pedersen, T. Gundersen, O. Hejlesen, Sensor acceptance model - measuring patient acceptance of wearable sensors, Methods Inf Med.

[16] J. Rincon, V. Julian, C. Carrascosa, Social Emotional Model, in: 13th International Conference on Practical Applications of Agents and Multi-Agent Systems, Vol. 9086 of LNAI, 2015, pp. 199-210.

[17] P. J. Lang, M. M. Bradley, B. N. Cuthbert, International affective picture system (IAPS): Affective ratings of pictures and instruction manual, Tech. Rep. A-8, The Center for Research in Psychophysiology, University of Florida, Gainesville, FL (2008).

[18] M. Bradley, P. J. Lang, Measuring Emotion: The Self-Assessment Semantic Differential Manikin and the, Journal of Behavior Therapy and Experimental Psychiatry 25 (I) (1994) 49-59. 